\title{
论文
}

\section{青藏高原末次冰消期狩猎采集人群的生存 策略研究}

\author{
王建 ${ }^{1}$ ，夏欢 ${ }^{1}$ ，姚娟婷 ${ }^{1}$ ，申旭科 ${ }^{1}$ ，成婷 ${ }^{1}$ ，王倩倩 ${ }^{2}$, 张东菊 ${ }^{*}$ \\ 1. 兰州大学资源环境学院, 兰州大学西部环境教育部重点实验室, 兰州 730000; \\ 2. 青海省文物考古研究所, 西宁 810007 \\ *通讯作者, E-mail: djzhang@1zu.edu.cn
}

收稿日期：2019-06-24; 收修改稿日期：2019-09-09; 接受日期：2019-09-25; 网络版发表日期：2019-11-28 国家自然科学基金项目(批准号：41771225和41620104007)、兰州大学中央高校基本科研业务费专项项目(编号：LZUJBKY-2016-254、 LZUJBKY-2016-279、LZUJBKY-2018-144)和国家留学基金管理委员会资助

摘要青藏高原史前狩猎采集人群生存策略研究是理解早期人类适应高海拔环境机制的关键. 迄今, 青藏高原上 具有埋藏地层和可靠测年结果的旧石器时代考古遗址数量有限，主要集中分布在高原东北部的青海湖盆地，且仅 有部分遗址出土的少量动植物遗存能够在一定程度上反映出古人类的生存策略. 151 遗址位于青海湖南岸, 在2014 年考古发掘中出土了较为丰富的动物骨骼遗存, 为研究史前狩猎采集人群在青藏高原的生存策略提供了重要材 料. 本文通过对151遗址下文化层(15400 13100 cal a BP)动物骨骼遗存的埋藏学和动物考古学分析, 并结合研究区 内其他同时期遗址已发表的研究成果，探讨青海湖盆地末次冰消期狩猎采集人群的生存模式. 动物骨骼遗存分析 结果显示, 151 遗址下文化层的狩猎采集人群主要狩猎大型有蹄类动物(野牛和野马/野驴), 并选择把营养价值较高 的猎物身体部位(上、中部肢骨、头部和躯干)搬运回遗址后, 围绕火塘对猎物进行处理和消费等. 对青海湖盆地内 末次冰消期遗址的综合分析结果显示，狩猎采集人群在盆地内是小规模群体的短期活动，采用对有蹄类动物的随 机狩猎策略和高移动的迁徙策略以适应高原环境. 此生存模式很可能是末次盛冰期之后，具有一定规模的狩猎采 集人群开始逐渐适应青藏高原极端环境的第一步，为全新世期间狩猎采集人群在高原上广泛分布奠定了基础. 因 此基于动物资源利用探讨青藏高原史前狩猎采集人群的生业模式，对深入理解史前人类向青藏高原的扩散并逐步 适应高海拔环境的过程和机制具有重要意义.

关键词青藏高原, 未次冰消期, 151遗址, 狩猎采集人群, 埋藏学, 动物考古学, 生存策略

\section{1 引言}

青藏高原由于其地理环境的极端性和特殊性，在
史前人地关系研究中具有重要意义, 特别是对于理解 人类如何适应高海拔环境尤为重要. 自 20 世纪70年代 考古学家开始在青藏高原开展系统的史前人类活动研 
究以来，青藏高原史前人类活动历史及其对高海拔环 境适应研究逐渐成为多学科关注的焦点(安志敏等, 1979；汤惠生，1999；Brantingham等，2003，2010; Brantingham和Gao, 2006; Madsen等, 2006; 袁宝印等, 2007; 高星等, 2008; Yi等, 2010; Huerta-Sánchez等, 2014; Chen等, 2015, 2019; 张东菊等, 2016; Meyer等, 2017; Zhang等, 2017, 2018; Zhang X L等, 2018). 然而, 由于缺乏有埋藏地层和可靠测年结果的旧石器时代遗 址，青藏高原上狩猎采集人群的活动历史长期以来无 法得以厘清. 近期发表的多项研究成果相继更新了我 们对史前人类占据青藏高原历史的认识，如尼阿底 (ND)遗址揭示现代人在距今 4 3万年前的末次冰期已 经在海拔 $4600 \mathrm{~m}$ 的高原腹地活动(Zhang X L等, 2018); 夏河人 $(\mathrm{XH})$ 下领骨化石的研究表明，丹尼索瓦人至少 在距今 16 万年前的倒数第二次冰期就已经生活在青藏 高原东北部，为现代青藏高原人群普遍具有的高海拔 环境适应基因—EPAS1(Endothelial PAS domaincontaining protein 1; Huerta-Sánchez等，2014)提供了 可能的本地来源, 表明丹尼索瓦人在现代人抵达之前 已经成功适应了高海拔环境(Chen等, 2019). 这两项研 究不仅极大地向前推进了史前人类在青藏高原活动的 时间, 而且为研究早期人类适应高寒缺氧环境提供了 更广阔的空间. 但是, 由于缺乏伴生的动植物遗存, 这 两项研究均未能回答早期人类如何在行为上适应高寒 缺氧和食物资源相对匮乏的高原环境的问题.

食物是人类生存的首要资源之一，食物资源利用 策略的研究是理解早期人类适应青藏高原环境的关 键. Chen等(2015)阐释了全新世中晚期的农牧业人群 在青藏高原的生业模式, 即农业和畜牧业为新石器-青 铜时代人群定居高海拔地区提供了可靠的食物来源. 然而, 在高原上出现农牧业之前, 狩猎采集人群只能依 赖野生动植物资源. 目前, 青藏高原上已知有可靠年代 的旧石器时代遗址主要集中于末次冰消期(Last Deglacial, 距今1.8 1.16万年), 且主要分布在青海湖盆地, 包 括江西沟1(JXG 1)、黑马河1(HMH 1)、93-13地点、 十火塘(10HTHS 1)、湖东种羊场(HZYC 1) 和铜线3 (BWC 3)等遗址(Madsen等，2006；仪明洁等，2011; Rhode等，2014)。该时期青藏高原逐渐转暖转湿的气 候可能是吸引低海拔地区狩猎采集人群频繁造访高原 边缘区域的主要原因之一(张东菊等, 2016). 上述遗址 的调查和试掘工作，为史前狩猎采集人群的高原生存
策略研究提供了初步材料(Madsen等，2006，2017; 仪 明洁等, 2011). 然而, 由于这些遗址均未经过正式的考 古发掘, 出土的动植物遗存数量非常有限, 不足以系统 地研究青藏高原史前狩猎采集人群生存策略.

本文基于青海湖盆地首次经正式考古发掘的史前 遗址一151遗址下文化层的动物骨骼材料, 应用AMS ${ }^{14} \mathrm{C}$ 测年方法建立可靠的地层年代, 通过系统的埋藏学 和动物考古学分析, 并结合盆地内其他同时期遗址的 已有研究成果, 探讨末次冰消期史前狩猎采集人群在 高原上的生存策略.

\section{2 遗址概况}

151 遗址 $\left(36.560^{\circ} \mathrm{N}, 100.475^{\circ} \mathrm{E}\right.$, 海拔 $\left.3397 \mathrm{~m}\right)$ 地处青 海省海南藏族自治州共和县江西沟乡151景区附近, 位 于青海湖南岸的青海南山北坡山前河流阶地上. 遗址 北距青海湖约 $3.5 \mathrm{~km}$, 高出青海湖现代湖面约 $203 \mathrm{~m}$, 西 侧有一条南北流向最终注入青海湖的小河沟(图1a和 1b). 151遗址由中美联合调查队发现于 2007 年(仪明洁 等，2011)，2014年青海省文物考古研究所和兰州大学 对其进行首次正式发掘, 发掘面积 $25 \mathrm{~m}^{2}(5 \mathrm{~m} \times 5 \mathrm{~m})$, 总发 掘深度约 $3.4 \mathrm{~m}$. 本次发掘除了揭露出在 2007 年调查中 发现的较厚的全新世早中期文化层(Rhode等，2014), 即本文的“上文化层”以外，在距地表约 $2.8 \mathrm{~m}$ 的探方西 北侧新发现一个文化层，即本文的“下文化层”(图1c). 上文化层与下文化层之间有约 $0.8 \mathrm{~m}$ 厚的自然黄土间歇 层. 本文仅分析下文化层出土的动物骨骼材料, 上文化 层将另撰文详述.

151遗址下文化层在2014年发掘探方中仅在靠近 西壁偏北约 $3 \mathrm{~m}^{2}$ 范围内出露, 厚约 $20 \mathrm{~cm}$, 主要由一个 单独的火塘及其周围灰烬区域组成，在火塘及灰烬区 域周围未发现临时性建筑遗迹(图1d). 火塘结构较简 单，大致呈椭圆形 $(90 \mathrm{~cm} \times 50 \mathrm{~cm})$ ，大量炭屑、火塘 石、石制品和动物骨骼集中分布其内, 火塘外也有零 星分布. 火塘石大小不一, 直径多在 $5 \sim 12 \mathrm{~cm}$, 多有角裂 现象, 表明曾经被火烧过. 石制品数量不多, 以中国北 方的小石片石器工业制品为主, 同时也有少量的细石 叶技术制品，工具较少. 石器技术和类型组合与青海 湖盆地内同时期的江西沟 1 和黑马河 1 等遗址相同 (Madsen等，2006). 动物骨骼数量相对较多，但非常 破碎. 

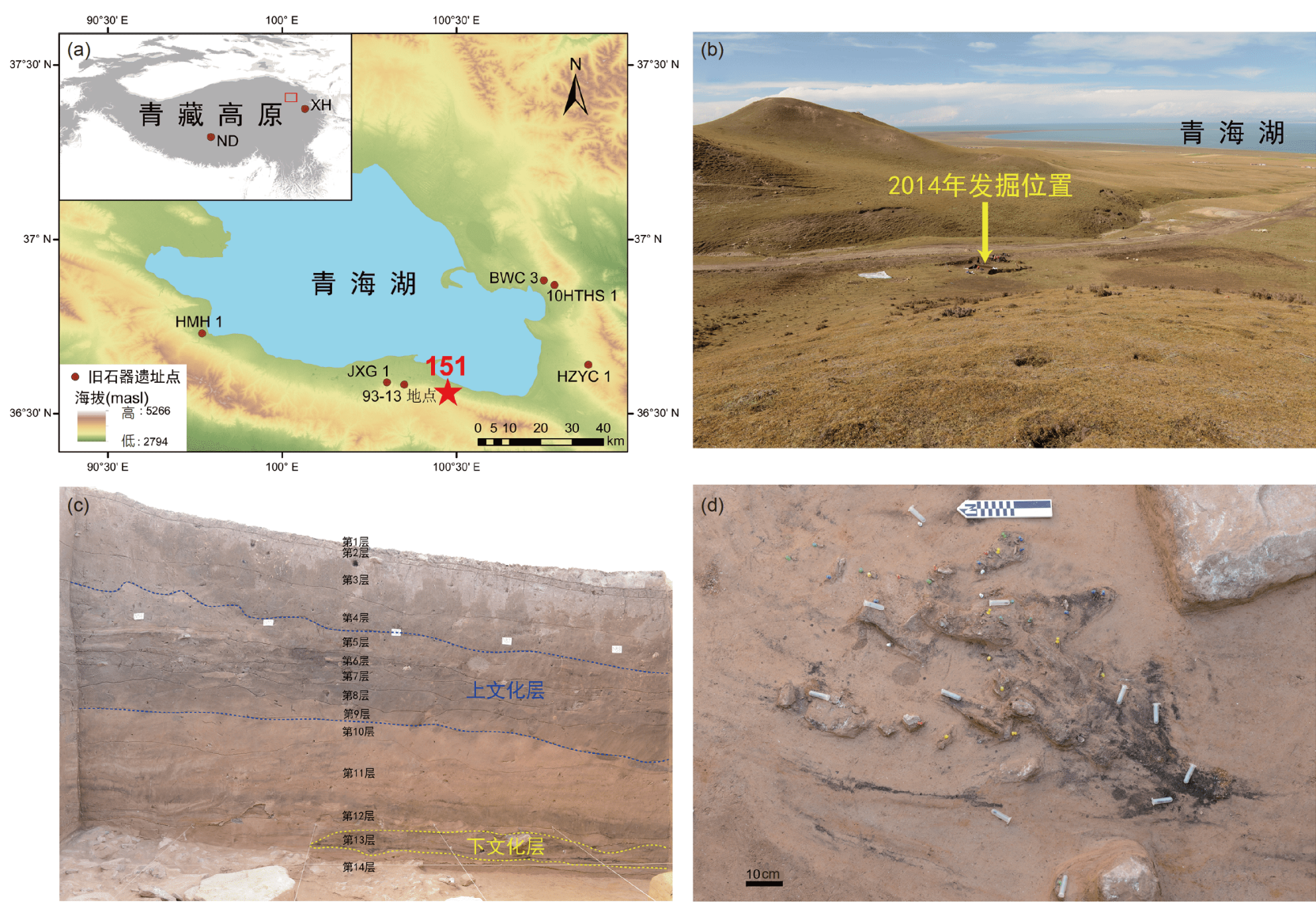

图 1 研究区及151遗址发掘概况

(a) 青海湖盆地末次冰消期旧石器时代遗址分布; (b) 151遗址2014年发掘位置; (c) 151遗址2014年发掘探方西壁剖面图; (d) 151遗址下文化层 火塘俯视图

\section{3 材料与方法}

151遗址下文化层的年代通过炭屑和动物骨骼的 AMS ${ }^{14} \mathrm{C}$ 测年结果建立，其动物骨骼遗存为本文的主 要研究材料.

\subsection{AMS ${ }^{14}$ 测年}

AMS ${ }^{14} \mathrm{C}$ 测年材料包括动物骨骼和炭屑. 动物骨 骼骨胶原的提取步骤参考 Ma等(2016), 炭屑样品的前 处理采用常规ABA(acid-base-acid, 酸-碱-酸)方法(Haesaerts等, 2013). 上述实验和后续石墨制备过程均在兰 州大学西部环境教育部重点实验室完成, 加速器测试 则在北京大学加速器质谱实验室完成. 本文涉及的全 部 ${ }^{14} \mathrm{C}$ 年代，都在 $\mathrm{OxCa}$ 软件中使用Intcal13树轮校正曲 线校正到日历年(Reimer等, 2013), 表述为cal a BP
(“BP”: 距公元1950年的时间).

\section{2 动物群分析方法}

在发掘过程中, 系统收集所有出土遗物. 对每一件 最大长度 $\geq 2 \mathrm{~cm}$ 的骨骼和 $<2 \mathrm{~cm}$ 有鉴定特征的骨骼都用 全站仪记录其三维坐标, 并在现场编号, 单独装袋收 集; 对每个发掘单元 $(1 \mathrm{~m} \times 1 \mathrm{~m} \times 0.05 \mathrm{~m})$ 的所有土样，用 $3 \mathrm{~mm}$ 篮网进行干篮和湿篮，收集所有的动植物和石制 品等遗存.

发掘结束后，在兰州大学西部环境教育部重点实 验室，先用 $10 \mathrm{~mm}$ 篎网对所有篮选获得的骨骼再次篮 选，然后根据实验室收藏的现代动物骨骼标本和动物 考古学资料，鉴定包括单独收集和篮选获得的所有可 鉴定骨骼. 本遗址由于动物骨骼极其破碎，因此大多 数可鉴定的动物骨骼被划分为动物体型大小而非具体 
的种属. 动物按体型大小被划分为大型有蹄类动物(如 野牦牛(Bos grunniens)和野马/野驴(Equus sp.))、中型 有蹄类动物(如马鹿(Cervus elaphus))、小型有蹄类动 物(如普氏原羚(Procapra przewalskii))和小型哺乳动物 (如野兔(Lepus sp.)和旱獭(Marmota sp.)).

在鉴定过程中, 鉴定所有可鉴定标本的骨骼, 包括 头骨、牙齿、椎骨、胁骨、四肢骨和指/趾骨等，并对 不可鉴定的 $\geq 1 \mathrm{~cm}$ 的骨骼进行计数. 根据骨骼标本的解 剖学特征确定、定量和记录其精确的骨骼单元或部位 及其完整率(Klein和Cruz-Uribe，1984；Lyman，1994). 在鉴定肢骨骨干碎片时，利用骨干的“特征部位”(diagnostic zones, Stiner, 2002)和其他形态学特征(Barba和 Domínguez-Rodrigo, 2005), 进行鉴定并量化其完整率. 根据牙齿萌出和磨损情况，分析野马/野驴的死亡年龄 (Fernandez和Legendre, 2003), 而野牛的牙齿因过于破 碎而无法用于年龄估计. 对动物群进行埋藏学分析, 了 解动物群形成和埋藏过程(Behrensmeyer, 1991; Lyman, 1994). 所有标本均在10倍手持放大镜下观察骨 骼表面痕迹, 包括人为痕迹(如切割痕迹、火烧痕迹和 敲击痕迹)、动物咬痕(食肉和啮齿动物咬痕)和其他自 然痕迹(如风化、植物根系腐蚀和踩踏痕迹等)(Behrensmeyer, 1978; Lyman, 1994; Blumenschine等, 1996). 同时记录长骨骨干的断裂状态(即在新鲜或干燥时断 裂)(Villa和Mahieu，1991)和长骨骨干周长的保存状况 (Marean等, 2004).

\section{4 结果}

\subsection{AMS ${ }^{14} \mathrm{C}$ 测年结果}

本文共获得 5 个AMS ${ }^{14} \mathrm{C}$ 年代，显示 151 遗址下文 化层的年代范围为15400 13100 cal a BP(表1), 与青 海湖盆地内的江西沟1(14200 14920cal a BP)、黑马 河1(12410 13440cal a BP)、湖东种羊场(12877 $13541 \mathrm{cal} \mathrm{a} \mathrm{BP})$ 等遗址年龄相近(Madsen等，2006, 2017). 151遗址下文化层的测年结果显示, 动物骨骼比 炭屑样品的年代要年轻几百年甚至一千多年，这符合 旧石器时代遗址中同层的动物骨骼样品通常比炭屑样 品更年轻的现象，可能与炭屑样品存在“老碳效应”有 关(Zilhão和d'Errico, 1999; Jöris等, 2003), 但也不排除 因多次人类活动导致文化层年代跨度较大的可能性.

\section{2 动物骨骼鉴定和分析结果}

151 遗址下文化层出土的动物骨骼总质量为 $445.9 \mathrm{~g}$, 共来自 23 个发掘单元. 动物群主要由极其破碎 的有蹄类动物骨干碎块和牙齿碎片组成, 动物种属主 要依据牙齿确定. 该层共出土 227 件最大长度 $\geq 1 \mathrm{~cm}$ 的 骨骼碎片和完整骨骼, 其中可鉴定标本数(NISP)为 126 件, 最小个体数(MNI)为 4 (表2). 151遗址下文化层的动 物骨骼数量虽然有限, 但这与大多数单个火塘遗址中 动物骨骼数量都非常少的现象相符(Vaquero和Pastó, 2001).

151遗址下文化层动物群种属组成相对简单, 主要 以大型有蹄类动物野牛 (Bos sp.) 和野马/野驴为主, 占 整个动物群的 $89.7 \%$; 其次为小型有蹄类动物 (普氏原 羚体型大小), 占整个动物群的 $9.5 \%$; 而小型哺乳动物 仅发现一件高原兔(Lepus oiostolus)的掌骨, 占整个动 物群的 $0.8 \%$ (表2). 值得注意的是，在动物群中没有发 现食肉动物骨骼. 151遗址下文化层与青海湖盆地内其 他同时期的江西沟 1 、黑马河 1 遗址均主要狩猎有蹄类 动物(Madsen等, 2006), 说明在中国其他某些区域发生 的“广谱革命”(张双权等, 2016)并未在末次冰消期的青 海湖盆发生. 关于动物的死亡年龄, 只有一件野马/野 驴的下领右侧第二前臼齿(p2)能提供有效信息，其牙 冠高度 $(20 \mathrm{~mm})$ 揭示该个体为壮年个体.

\section{5 讨论}

尽管151遗址下文化层出土的动物骨骼遗存数量 有限, 但为考古发掘过程中的系统收集, 具有较好的代 表性. 对这些动物骨骼遗存分析, 有助于对末次冰消期 青藏高原旧石器时代遗址的形成过程、史前人类的动 物资源利用策略和环境适应方式等问题的探讨.

\subsection{1 遗址动物群的埋藏学分析}

在对考古遗址中动物群的沉积历史和古人类生存 行为的研究中, 动物群的埋藏学分析发挥着重要作用. 考古遗址动物群在从生物圈的生活群转化成岩石圈的 化石群的过程中, 除了会受到多种自然因素的影响, 如 风化、分解、溶解、压实及石化等磨耗过程和食肉动 物、啮齿动物、猛禽的消费、搬运和啃咬等自然作 用，还会受到古人类对动物的获取、搬运、处理和消 
表 1 151遗址下文化层AMS ${ }^{14}$ C测年结果

\begin{tabular}{ccccc}
\hline \multirow{2}{*}{ 实验室编号 } & 测年材料 & ${ }^{14} \mathrm{C}$ 年代(a BP) & \multicolumn{2}{c}{ 校正年代(cal a BP) } \\
\cline { 4 - 5 } & & & $1 \delta$ & $15420 \pm 105$ \\
LZU14284 & 炭屑 & $12920 \pm 35$ & $14159.5 \pm 67.5$ & $14188.5 \pm 162.5$ \\
LZU16244 & 炭屑 & $12265 \pm 35$ & $14040 \pm 65$ & $14010.5 \pm 153.5$ \\
LZU14285 & 炭屑 & $12150 \pm 40$ & $13699 \pm 72$ & $13680 \pm 101$ \\
LZU16291 & 动物骨骼 & $11895 \pm 35$ & $13142.5 \pm 45.5$ & $13159 \pm 89$ \\
LZU16292 & 动物骨骼 & $11300 \pm 40$ & & \\
\hline
\end{tabular}

表 2 151遗址下文化层动物群中可鉴定标本的骨骼单元分布表 ${ }^{a)}$

\begin{tabular}{|c|c|c|c|c|c|c|}
\hline & 骨骼单元 & 野牛 & 野马/野驴 & 大型有蹄类动物 & 小型有蹄类动物 & 高原兔 \\
\hline \multirow{4}{*}{ 头部 } & 头骨 & & & 1 & & \\
\hline & 上领 & & & 1 & & \\
\hline & 下领骨 & & & 3 & 1 & \\
\hline & 下领牙齿 & 1 & 3 & & & \\
\hline \multirow{3}{*}{ 躯干骨 } & 颈椎 & & & 1 & & \\
\hline & 椎骨 & & & & 1 & \\
\hline & 肋骨 & & & 20 & 5 & \\
\hline \multirow{5}{*}{ 前肢骨 } & 肩胛骨 & & & & & \\
\hline & 肱骨, 近端/远端/骨干 & $0 / 0 / 2$ & & $0 / 0 / 1$ & & \\
\hline & 桡骨, 近端/远端/骨干 & & & $0 / 0 / 1$ & & \\
\hline & 尺骨, 近端/远端/骨干 & & & & & \\
\hline & 掌骨, 近端/远端/骨干 & & & & & $1^{*}$ \\
\hline \multirow{4}{*}{ 后肢骨 } & 骹骨 & & & & 1 & \\
\hline & 股骨, 近端/远端/骨干 & & & & & \\
\hline & 胫骨, 近端/远端/骨干 & & & $0 / 0 / 2$ & $0 / 0 / 1$ & \\
\hline & 跖骨, 近端/远端/骨干 & & & & & \\
\hline \multirow{6}{*}{ 指/趾骨 } & 指/趾骨III, 近端/远端 & & & & $1 / 0$ & \\
\hline & 不可鉴定, 牙齿碎片 & & & 34 & & \\
\hline & 不可鉴定, 长骨 & & & 43 & 3 & \\
\hline & NISP & 3 & 3 & 107 & 12 & 1 \\
\hline & MNI & 1 & 1 & & 1 & 1 \\
\hline & $\% \mathrm{NISP}$ & 2.4 & 2.4 & 84.9 & 9.5 & 0.8 \\
\hline
\end{tabular}

a) 大型有蹄类动物包括野牛和野马/野驴, 小型有蹄类动物指普氏原羚体型大小的有蹄类动物, *表示完整的骨骼

费等人为作用的影响(Lyman, 1994). 因此, 考古遗址中 动物群的形成往往是一个复杂而综合的过程，在对其 所反映的人类行为分析之前, 需要首先对动物群的埋 藏过程进行分析.

151遗址下文化层动物群以有蹄类动物为主和以 单个火塘为人类活动中心的现象，能够在一定程度上
排除啮齿动物(Brain，1981)和猛禽(Andrews，1990)对 动物骨骼聚集的可能性; 动物骨骼表面缺少食肉和啮 齿动物的改造(表3)和消化痕迹，表明食肉动物、啮齿 动物和猛禽并未参与动物骨骼的聚集. 由于遗址位于 山前坡地上，坡面流水作用似乎更能影响遗址动物群 的聚集. 对于一些遗址动物群而言，流水作用更多的 
表 3 151遗址下文化层有蹄类动物骨骼表面痕迹和骨骼断裂模式统计表 ${ }^{a)}$

\begin{tabular}{|c|c|c|c|}
\hline \multicolumn{2}{|c|}{ 骨骼表面痕迹和骨骼断裂模式 } & 大型有蹄类动物 & 小型有蹄类动物 \\
\hline \multirow{2}{*}{ 烧骨 } & 个数 $(n)$ & 2 & 7 \\
\hline & 百分比(\%) & 2.7 & 58.3 \\
\hline 新鲜断裂 & 个数 $(n)$ & 30 & 1 \\
\hline 干燥断裂 & 个数 $(n)$ & 14 & 0 \\
\hline 中间状态 & 个数 $(n)$ & 1 & 0 \\
\hline \multirow{3}{*}{ 长骨骨干周长 } & $<50 \%$ & 45 & 2 \\
\hline & $>50 \%$ & 0 & 0 \\
\hline & $100 \%$ & 0 & 0 \\
\hline \multirow{2}{*}{ 风化等级 (3 5级) } & 个数 $(n)$ & 68 & 2 \\
\hline & 百分比(\%) & 90.7 & 16.7 \\
\hline \multirow{2}{*}{ 切割痕迹 } & 个数 $(n)$ & 2 & 1 \\
\hline & 百分比(\%) & 2.7 & 8.3 \\
\hline \multirow{2}{*}{ 敲击痕迹 } & 个数 $(n)$ & 0 & 0 \\
\hline & 百分比(\%) & 0 & 0 \\
\hline \multirow{2}{*}{ 食肉动物啃咬痕迹 } & 个数 $(n)$ & 0 & 0 \\
\hline & 百分比(\%) & 0 & 0 \\
\hline \multirow{2}{*}{ 啮齿动物磨牙痕迹 } & 个数 $(n)$ & 0 & 0 \\
\hline & 百分比(\%) & 0 & 0 \\
\hline \multirow{2}{*}{ 植物根系腐蚀痕迹 } & 个数 $(n)$ & 4 & 1 \\
\hline & 百分比(\%) & 5.3 & 8.3 \\
\hline \multirow{2}{*}{ 踩踏痕迹 } & 个数 $(n)$ & 0 & 0 \\
\hline & 百分比(\%) & 0 & 0 \\
\hline
\end{tabular}

a) 有蹄类动物骨骼表面改造和骨骼断裂模式的统计不包括牙齿和牙齿碎片, 其中大型有蹄类动物的标本总数为 75 , 小型有蹄类动物的 标本总数为 12 .

是改造已有的动物组合而非聚集动物骨骼(Behrensmeyer, 1978). 151遗址下文化层中 $23.78 \%(\mathrm{NISP}=29)$ 的 动物骨骼表面有水流磨蚀痕迹，同时火塘及其周围大 量炭屑均呈现出类似弱流水作用下沿坡面的带状分布 (图1d)，表明存在一定的流水改造作用. 然而，石制品 和动物骨骼总体上以火塘为中心分布，并无明显的定 向分布现象(图1d), 说明主要的考古遗存原生位置未 发生改变，该文化层是古人类人为作用影响下的原生 堆积.

\subsection{1 遗址的古人类生存行为分析}

火塘是构成狩猎采集人群社会单元凝聚力的基 础, 是人类活动的核心区域, 也是史前和现代狩猎采集 人群主要的空间利用特征(O'Connell, 1987; O'Connell 等, 1991; Vaquero和Pastó, 2001). 早在旧石器时代中
期，狩猎采集人群就围绕火塘开展一系列对食物的处 理、分享以及工具的生产和维护等活动(Stiner等, 2009). 火塘的大小能在一定程度上反映人口规模，而 狩猎采集人群的人口规模则是理解古人类生存行为的 重要前提之一. 当人们围绕火塘进行一系列的消费和 生产等活动时, 个体需要占有一定的活动空间, 火塘 的大小会限制群体的规模，因而考古遗址中火塘遗迹 的大小可以反映古人类的人口规模. 当人们围绕火塘 活动时, 主要的活动范围是以火塘中心为圆心、半径 约 $1.76 \mathrm{~m}$ 左右的区域(Binford，1996)，通常一个个体所 能波及的活动范围约 2.5 3 $\mathrm{m}^{2}$ (Freeman, 1978), 如果将 其视为一个圆形区域，其直径为 $1.80 \sim 1.94 \mathrm{~m}$ (Henry, 2012). 基于上述理论能够推算出, 151遗址下文化层单 个直径约 $1 \mathrm{~m}$ 的火塘，适合 $1 \sim 5$ 个人的家庭规模的群体 活动, 若超过 5 个人就会过度拥挤. 此外, 江西沟 1 、黑 
马河 1及93-13地点等遗址的单个或分散的火塘直径也 都在0.5 1.5m(Madsen等, 2006，2017)，均小于现代狩 猎采集社会中较大群体的火塘，而与现代狩猎采集人 群短期营地的火塘范围相符(Yellen, 1977). 因此, 青海 湖盆地内多个末次冰消期遗址的火塘大小分析显示, 该时期的狩猎采集人口规模非常有限(可能以家庭为 单位), 并且都以短期活动为主.

动物骨骼单元分布分析可为古人类获取、处理、 搬运猎物等行为提供重要信息. 151遗址下文化层小型 有蹄类动物的材料非常有限 $(\mathrm{NISP}=12)$, 难以分析其搬 运策略; 大型有蹄类动物(野牛和野马/野驴)的骨骼单 元分布(表2)表明，151遗址的古人类可能对大型猎物 的身体部位进行有选择性的搬运，即把营养价值较高 的上、中部肢骨(股骨、肱骨、胫骨和桡尺骨)和躯干 (椎骨和肋骨)搬运回遗址进一步处理和消费. 大型有 蹄类动物(如野牛)体型巨大，古人类一般不能完整地 把猎物搬运回营地，常常在狩猎地槑掉动物的角、取 出内脏并将其肢解成若干大的解剖单元(通常单独肢 解下四肢)，然后再选择某些特定的部分搬运(Bunn等, 1986). 虽然头部和躯干骨(椎骨和肋骨)的标准营养效 用指数(standardized food utility index)相对四肢骨而 言不高(Lyman, 1994), 但是对于古人类而言, 即使在狩 猎地已对其进行了初步的肌肉和骨髓提取，仍然会有 大量营养物质残留在骨骼上(Marean和Cleghorn, 2003). 151遗址下文化层大型有蹄类动物(野牛和野马/ 野驴)的上下领牙齿、颈椎和大量肋骨碎片的出现可 能表明, 为了避免头骨和躯干部位营养物质的浪费, 狩 猎采集人群选择将这些部位带回营地进行更加细致和 彻底的营养物质提取. 这与民族学上观察到的Hadza猎 人在营养物质最大化与搬运成本最小化(即骨骼干重) 之间维持平衡和折衷的搬运现象相似(Lupo, 2006).

猎人搬运猎物并不是一件轻松的工作。尽管单个 猎人最多能搬运 $25 \sim 30 \mathrm{~kg}$ 的猎物，但要将一根 $35 \sim 40 \mathrm{~kg}$ 重的完整且包括附属组织的水牛大腿运回约 $5 \mathrm{~km}$ 之外 的营地, 往往需要多达 24 人的团队合作, 且在搬运途中 需要多次休息和补充能量(Hall，2007). 野马的体型相 对于水牛明显要小一些，但搬运仍然需要由几个猎人 合作完成(Binforn, 1978; Bunn, 1993). 显然, 只有当搬 运距离很远或者参与人数特别少时，搬运这些大型动 物才需要猎人做出是仅带回较高营养价值的部位还是 全部带回的取舍. 而在群体人口规模有限的情况下,
151遗址古人类选择将头骨和躯干也搬回遗址，说明 狩猎地可能距151遗址并不远. 此外, 野牛和野马/野驴 对水源高度依赖的生理特征与 151 遗址邻近小河的环 境特征, 显示古人类可能就在遗址附近狩猎.

动物骨骼表面痕迹和断裂模式也能够提供关于古 人类行为方面的重要信息. 151 遗址下文化层动物骨骼 表面痕迹分析结果表明，大型有蹄类动物骨骼在沉积 前的风化程度远高于小型有蹄类动物(表3). 这可能是 由于更大体积的大型有蹄类动物骨骼被堆积物完全掩 埋需要更长的时间, 因此风化程度会更高. 当然也可能 是古人类对遗址多次利用的结果，即在不同的狩猎事 件后把大型和小型有蹄类动物分别带回遗址. 此外, 动物骨骼表面风化严重，也不利于进行古人类对猎物 的处理和消费等行为分析. 对有蹄类动物长骨骨干的 断裂模式(断口形态和断口质地)分析(Villa和Mahieu, 1991)显示，151遗址下文化层长骨骨干以新鲜断裂为 主(表3), 表明可能存在敲骨吸髓行为. 此外, 151遗址 下文化层中存在火裂石，动物骨骼以长骨骨干碎片为 主，长骨骨骺部分多缺失和破碎(表2)，暗示古人类可 能存在通过“热石煮沸”法(hot rock boiling)(Lupo, 1995)来获得附着在骨骼内及骨骺端残存油脂等营养 物质的行为(Madsen等, 2006). 因为能量效率高和热量 值高的动物油脂在饮食结构中起着重要作用，尤其对 于哺乳期妇女和儿童尤为重要(Burger等, 2005), 而且 油脂和密封在油脂中的肉类比新鲜的肉和干肉能保存 更久(Vehik, 1977). 此外, 江西沟1和黑马河1遗址的动 物群同样也都是以有蹄类动物的破碎肢骨为主，且都 存在火裂石(Madsen等, 2006). 虽然目前还不能确定盆 地内这些遗址是否都存在提取骨骼中油脂的行为，但 普遍存在的骨骼破碎的现象, 说明末次冰消期盆地内 的古人类为应对高原环境中高能量消耗需求，采取了 同样的动物资源强化利用策略.

\section{3 青藏高原史前狩猎采集人群的环境适应策略}

151遗址下文化层和青海湖盆地内其他末次冰消 期的江西沟 1 、黑马河 1 遗址都以狩猎有蹄类动物为 主，但在猎物组成上有着显著的不同. 151遗址下文化 层主要以大型有蹄类动物为主，而江西沟 1 和黑马河 1 遗址以羚羊体型大小的有蹄类动物为主(Madsen等, 2006). 最佳受食理论(optimal foraging theory, Smith等, 1983)的食谱宽度模型(dietary breadth models)显示, 人 
类在受食过程中与其他动物一样, 都期望能最大限度 地获取能量, 即首选能量最多且回报率最高的动物(如 有蹄类动物), 或是捕获成本较低的动物(如乌龟)(Winterhalder, 1986). 末次冰消期青海湖盆地内的各个遗址 都以高回报率的有蹄类动物为主, 考虑到当人类短期 占据某一遗址时, 不太可能在短时间占据期间消耗尽 所有较高回报率的动物(Stiner, 2002). 因此, 我们认为 青海湖盆地的狩猎人群在遵循最佳受食理论的前提 下, 对高回报率的有蹄类动物进行随机狩猎(Bunn, 2001), 以满足小规模人群在不同遗址内短期生活的需 求. 当然, 也不能排除在同样技术条件下不同人群不同 生存策略的可能性. 此外, 同时期多个短期营地的出 现, 说明末次冰消期狩猎采集人群在青海湖盆地采用 了高移动的迁徙策略(Kelly等, 2005).

短期、小规模的狩猎采集人群在末次冰消期出现 在青海湖盆地可能得益于逐渐转湿转暖的气候环境与 狩猎技术的革新. 一方面, 在末次盛冰期之后, 青海湖 盆地温度增高和降水增多(Yu和Kelts, 2002; Colman等, 2007), 植被类型由荒漠草原向高山草甸/亚高山灌丛 过渡(Shen等, 2005), 动物种群得以恢复, 这些变化为 古人类在盆地内生活提供了更多的地理空间和食物资 源. 另一方面, 新的石器技术一细石叶技术开始出现 在高原上, 细石叶通常被认为与恶劣环境中高迁移性 的狩猎活动密切相关, 也是更安全地狩猎大型动物、 制作复杂的兽皮或毛皮服装以及用植物纤维加工缝㧅 的有效工具(Yi等, 2016). 细石叶技术的这些优势有助 于应对资源相对贵乏、昼夜温差大等不利人类生存的 高原环境. 此外, 青藏高原季节性气候变化显著, 资源 斑块质量时空变化起伏大, 季节性资源易预估意味着 较高的狩猎成功率, 从而对狩猎采集人群更有吸引力. 但是, 显著的季节性差异也意味着在冬春季节资源更 加贵乏和气候更为恶劣(时兴合等, 2006), 从而导致狩 猎采集人群不得不迁徙到低海拔区域，从而无法常年 占据高原(张东菊等, 2016; Zhang等, 2017).

\section{6 结论}

青海湖盆地151遗址下文化层的发现, 丰富了末次 冰消期狩猎采集人群在高海拔地区活动的证据. 151遗 址出土的动物骨骼材料的埋藏学和动物考古学分析, 揭示了该遗址史前狩猎采集人群对动物资源的搬运和
利用策略: 对大型有蹄类动物, 古人类选择将营养价值 较高的上、中部肢骨、头部和躯等部位搬运回遗址 进行处理和消费; 在资源利用上, 古人类除了消费肉类 资源外, 还可能很注重对骨髓甚至骨骼油脂的利用, 以 适应高海拔环境中对高能量消耗的需求. 通过与青海 湖盆地其他同时期遗址进行对比分析, 发现盆地内普 遍存在由单个或分散火塘遗迹构成遗址的现象，且不 同遗址的古人类主要狩猎不同体型有蹄类动物. 综合 分析显示, 末次冰消期短期占据青海湖盆地的小规模 狩猎采集人群随机狩猎高回报率的有蹄类动物, 并采 用高频率迁徙策略和可能的动物资源强化利用策略, 以适应高能量需求和季节性显著的高原环境.

致谢感谢崔一付、黎海明、范文洁、刘雨嘉、刘小慢 等同学在野外发掘和材料整理过程中给予的帮助, 国家 文物局和青海省共和县文物体育广播电视局对野外发掘 工作的大力支持, 山东大学历史文化学院遗产研究院(青 岛)的王华副教授、德国Max Planck Institute for Evolutionary Anthropology的Geoffrey Michael Smith研究员和 兰州大学历史文化学院任乐乐博士在动物种属鉴定中给 予的指导和帮助, 美国Wesleyan University的Katherine Brunson博士提供的有益修改建议.

\section{参考文献}

安志敏, 尹泽生, 李炳元. 1979. 藏北申扎、双湖的旧石器和细石器. 考古, (6): 481-491

高星, 周振宇, 关莹. 2008. 青藏高原边缘地区晚更新世人类遗存与 生存模式. 第四纪研究, 28: 969-977

时兴合, 李风霞, 扎西才让, 郭安红, 达成荣, 唐红玉. 2006. 1961 2004年青海积雪变化. 应用气象学报, 17: 376-382

汤惠生. 1999. 略论青藏高原的旧石器和细石器. 考古, (5): 428-438 仪明洁, 高星, 张晓凌, 孙永娟, Brantingham P J, Madsen D B, Rhode D. 2011. 青藏高原边缘地区史前遗址2009年调查试掘报告. 人类 学学报, 30: 124-136

袁宝印, 黄慰文, 章典. 2007. 藏北高原晚更新世人类活动的新证据. 科学通报, 52: 1567-1571

张东菊, 董广辉, 王辉, 任晓燕, 哈比布, 强明瑞, 陈发虎. 2016. 史前 人类向青藏高原扩散的历史过程和可能驱动机制. 中国科学: 地 球科学, 46: 1007-1023

张双权, 张乐, 栗静舒, 高星. 2016. 晚更新世晚期中国古人类的广谱 适应生存一一动物考古学的证据. 中国科学: 地球科学, 46 : 1024-1036

Andrews P. 1990. Owls, Caves and Fossils: Predation, Preservation, and 
Accumulation of Small Mammal Bones in Caves, with an Analysis of the Pleistocene Cave Faunas from Westbury-Sub-Mendip, Somerset, UK. Chicago: University of Chicago Press

Barba R, Domínguez-Rodrigo M. 2005. The taphonomic relevance of the analysis of bovid long limb bone shaft features and their application to element identification. J Taphonomy, 3: 17-42

Behrensmeyer A K. 1978. Taphonomic and ecologic information from bone weathering. Paleobiology, 4: 150-162

Behrensmeyer A K. 1991. Terrestrial vertebrate accumulations. In: Allison P A, Briggs D E G, eds. Taphonomy: Releasing the Data Locked in the Fossil Record. New York: Plenum Press. 291-335

Binford L R. 1978. Nunamiut Ethnoarchaeology. New York: Academic Press

Binford L R. 1996. Hearth and home, the spatial analysis of ethnographically documented rock shelter occupations as a template for distinguishing between human and hominid use of sheltered space. In: Conard N, Wendorf F, eds. Middle Paleolithic and Middle Stone Age Settlement Systems. Forlí: A.B.A.C.O Edizioni. 229-239

Blumenschine R J, Marean C W, Capaldo S D. 1996. Blind tests of inter-analyst correspondence and accuracy in the identification of cut marks, percussion marks, and carnivore tooth marks on bone surfaces. J Archaeol Sci, 23: 493-507

Brain C K. 1981. The Hunters or the Hunted? Chicago: University of Chicago Press

Brantingham P J, Ma H Z, Olsen J W, Gao X, Madsen D B, Rhode D E. 2003. Speculation on the timing and nature of Late Pleistocene hunter-gatherer colonization of the Tibetan Plateau. Chin Sci Bull, 48: $1510-1516$

Brantingham P J, Gao X. 2006. Peopling of the northern Tibetan Plateau. World Archaeol, 38: 387-414

Brantingham P J, Rhode D, Madsen D B. 2010. Archaeology augments Tibet's genetic history. Science, 329: 1467

Bunn H T, Kroll E M, Ambrose S H, Behrensmeyer A K, Binford L R, Blumenschine R J, Klein R G, McHenry H M, O’Brien C J, Wymer J J. 1986. Systematic butchery by Plio/Pleistocene hominids at Olduvai Gorge, Tanzania (and comments and reply). Curr Anthropol, 27: 431-452

Bunn H T. 1993. Bone assemblages at base camps: A further consideration of carcass transport and bone destruction by the Hadza. In: Hudson J, ed. From Bones to Behavior: Ethnoarchaeological and Experimental Contributions to the Interpretation of Faunal Remains. Carbonadale: Center for Archaeological Investigations. $156-168$

Bunn H T. 2001. Hunting, power scavenging, and butchering by Hadza foragers and by Plio-Pleistocene Homo. In: Stanford C B, Bunn H T, eds. Meat-eating and Human Evolution. Oxford: Oxford University
Press. 199-218

Burger O, Hamilton M J, Walker R. 2005. The prey as patch model: Optimal handling of resources with diminishing returns. J Archaeol Sci, 32: $1147-1158$

Chen F H, Dong G H, Zhang D J, Liu X Y, Jia X, An C B, Ma M M, Xie Y W, Barton L, Ren X Y, Zhao Z J, Wu X H, Jones M K. 2015. Agriculture facilitated permanent human occupation of the Tibetan Plateau after 3600 B.P. Science, 347: 248-250

Chen F H, Welker F, Shen C C, Bailey S E, Bergmann I, Davis S, Xia H, Wang H, Fischer R, Freidline S E, Yu T L, Skinner M M, Stelzer S, Dong G R, Fu Q M, Dong G H, Wang J, Zhang D J, Hublin J J. 2019. A late Middle Pleistocene Denisovan mandible from the Tibetan Plateau. Nature, 569: 409-412

Colman S M, Yu S Y, An Z, Shen J, Henderson A C G. 2007. Late Cenozoic climate changes in China's western interior: A review of research on lake Qinghai and comparison with other records. Quat Sci Rev, 26: 2281-2300

Fernandez P, Legendre S. 2003. Mortality curves for horses from the Middle Palaeolithic site of Bau de l'Aubesier (Vaucluse, France): Methodological, palaeo-ethnological, and palaeo-ecological approaches. J Archaeol Sci, 30: 1577-1598

Freeman L. 1978. Mousterian worked bone from Cueva Morin (Santander, Spain), a preliminary description. In: Freeman L G, ed. Views of the Past: Essays in Old World Prehistory and Paleoanthropology. Chicago: Aldine. 29-52

Haesaerts P, Damblon F, Nigst P, Hublin J J. 2013. ABA and ABOx radiocarbon cross-dating on charcoal from Middle Pleniglacial loess deposits in Austria, Moravia, and Western Ukraine. Radiocarbon, 55: $641-647$

Hall J N. 2007. Late prehistoric (Oneota) exploitation of bison, elk, and deer at the Howard Goodhue site, central Iowa. Dissertation for Master's Degree. Iowa: University of Iowa

Henry D. 2012. The palimpsest problem, hearth pattern analysis, and Middle Paleolithic site structure. Quat Int, 247: 246-266

Huerta-Sánchez E, Jin X, Asan X, Bianba Z, Peter B M, Vinckenbosch N, Liang Y, Yi X, He M, Somel M, Ni P, Wang B, Ou X, Huasang X, Luosang J, Cuo Z X P, Li K, Gao G, Yin Y, Wang W, Zhang X, Xu X, Yang H, Li Y, Wang J, Wang J, Nielsen R. 2014. Altitude adaptation in Tibetans caused by introgression of Denisovan-like DNA. Nature, 512: 194-197

Jöris O, Fernandez E, Weninger B. 2003. Radiocarbon evidence of the Middle to Upper Paleolithic transition in southwestern Europe. Trabajos Prehistoria, 60: 15-38

Kelly R L, Poyer L, Tucker B. 2005. An ethnoarchaeological study of mobility, architectural investment, and food sharing among Madagascar's Mikea. Am Anthropol, 107: 403-416 
Klein R G, Cruz-Uribe K. 1984. The Analysis of Animal Bones from Archaeological Sites. Chicago: Chicago University Press

Lupo K D. 1995. Hadza bone assemblages and hyena attrition: An ethnographic example of the influence of cooking and mode of discard on the intensity of scavenger ravaging. J Anthropol Archaeol, 14: 288-314

Lupo K D. 2006. What explains the carcass field processing and transport decisions of contemporary hunter-gatherers? Measures of economic anatomy and zooarchaeological skeletal part representation. J Archaeol Method Theor, 13: 19-66

Lyman L R. 1994. Vertebrate Taphonomy. Cambridge: Cambridge University Press

Ma M M, Dong G H, Jia X, Wang H, Cui Y F, Chen F H. 2016. Dietary shift after $3600 \mathrm{cal}$ yr BP and its influencing factors in northwestern China: Evidence from stable isotopes. Quat Sci Rev, 145: 57-70

Madsen D B, Ma H Z, Brantingham P J, Xing G, Rhode D, Haiying Z, Olsen J W. 2006. The Late Upper Paleolithic occupation of the northern Tibetan Plateau margin. J Archaeol Sci, 33: 1433-1444

Madsen D B, Perreault C, Rhode D, Sun Y J, Yi M J, Brunson K, Brantingham P J. 2017. Early foraging settlement of the Tibetan Plateau highlands. Archaeol Res Asia, 11: 15-26

Marean C W, Cleghorn N. 2003. Large mammal skeletal element transport: Applying foraging theory in a complex taphonomic system. J Taphonomy, 1: 15-42

Marean C W, Domínguez-Rodrigo M, Pickering T R. 2004. Skeletal element equifinality in zooarchaeology begins with method: The evolution and status of the "shaft critique". J Taphonomy, 2: 69-98

Meyer M C, Aldenderfer M S, Wang Z, Hoffmann D L, Dahl J A, Degering D, Haas W R, Schlütz F. 2017. Permanent human occupation of the central Tibetan Plateau in the early Holocene. Science, 355: 64-67

O'Connell J F. 1987. Alyawara site structure and its archaeological implications. Am Antiq, 52: 74-108

O’Connell J F, Hawkes K, Jones B N. 1991. Distribution of refuseproducing activities at Hadza residential base camps: Implications for analyses of archaeological site structure. In: Kroll E M, Price T D, eds. The Interpretation of Archaeological Spatial Patterning. New York: Plenum Press. 61-76

Reimer P J, Baillie M G L, Bard E, Bayliss A, Beck J W, Blackwell P G, Bronk Ramsey C, Buck C E, Burr G S, Edwards R L, Friedrich M, Grootes P M, Guilderson T P, Hajdas I, Heaton T J, Hogg A G, Hughen K A, Kaiser K F, Kromer B, McCormac F G, Manning S W, Reimer R W, Richards D A, Southon J R, Talamo S, Turney C S M, van der Plicht J, Weyhenmeyer C E. 2013. IntCal09 and Marine09 radiocarbon age calibration curves, 0-50000 years cal BP. Radiocarbon, 51: 1111-1150
Rhode D, Brantingham P J, Perreault C, Madsen D B. 2014. Mind the gaps: Testing for hiatuses in regional radiocarbon date sequences. $\mathrm{J}$ Archaeol Sci, 52: 567-577

Shen J, Liu X Q, Wang S M, Matsumoto R. 2005. Palaeoclimatic changes in the Qinghai Lake area during the last 18000 years. Quat Int, 136: 131-140

Smith E A, Bettinger R L, Bishop C A, Blundell V, Cashdan E, Casimir M J, Christenson A L, Cox B, Dyson-Hudson R, Hayden B, Richerson P J, Roth E A, Simms S R, Stini W A. 1983. Anthropological applications of optimal foraging theory: A critical review [and comments and reply]. Curr Anthropol, 24: 625-651

Stiner M C. 2002. On in situ attrition and vertebrate body part profiles. J Archaeol Sci, 29: 979-991

Stiner M C, Barkai R, Gopher A. 2009. Cooperative hunting and meat sharing 400-200 kyr at Qesem Cave, Israel. Proc Natl Acad Sci USA, 106: 13207-13212

Vaquero M, Pastó I. 2001. The definition of spatial units in Middle Palaeolithic sites: The hearth-related assemblages. J Archaeol Sci, 28: $1209-1220$

Vehik S C. 1977. Bone fragments and bone grease manufacturing: A review of their archaeological use and potential. Plains Anthropol, 22: 169-182

Villa P, Mahieu E. 1991. Breakage patterns of human long bones. J Human Evol, 21: 27-48

Winterhalder B. 1986. Diet choice, risk, and food sharing in a stochastic environment. J Anthropol Archaeol, 5: 369-392

Yellen J E. 1977. Archaeological Approaches to the Present. New York: Academic Press

Yi M J, Gao X, Li F, Chen F Y. 2016. Rethinking the origin of microblade technology: A chronological and ecological perspective. Quat Int, 400: 130-139

Yi X, Liang Y, Huerta-Sanchez E, Jin X, Cuo Z X P, Pool J E, Xu X, Jiang H, Vinckenbosch N, Korneliussen T S, Zheng H C, Liu T, He W M, Li K, Luo R B, Nie X F, Wu H L, Zhao M R, Cao H Z, Zou J, Shan Y, Li S Z, Yang Q, AsanNi P X, Tian G, Xu J M, Liu X A, Jiang T, Wu R H, Zhou G Y, Tang M F, Qin J J, Wang T, Feng S J, Li G H, HuasangLuosang J B, Wang W, Chen F, Wang Y D, Zheng X G, Li Z, Bianba Z M, Yang G, Wang X P, Tang S H, Gao G Y, Chen Y, Luo Z, Gusang L, Cao Z, Zhang Q H, Ouyang W H, Ren X L, Liang H Q, Zheng H S, Huang Y B, Li J X, Bolund L, Kristiansen K, Li Y R, Zhang Y, Zhang X Q, Li R Q, Li S G, Yang H M, Nielsen R, Wang J, Wang J A. 2010. Sequencing of 50 human exomes reveals adaptation to high altitude. Science, 329: 75-78

Yu J Q, Kelts K R. 2002. Abrupt changes in climatic conditions across the late-glacial/Holocene transition on the N. E. Tibet-Qinghai Plateau: Evidence from Lake Qinghai, China. J Paleolimnol, 28: 
195-206

Zhang D J, Zhang N M, Wang J, Ha B B, Dong G H, Chen F H. 2017.

Comment on "Permanent human occupation of the central Tibetan Plateau in the early Holocene". Science, 357: eaam8273

Zhang D J, Xia H, Chen F H. 2018. Early human occupation of the Tibetan Plateau. Chin Sci Bull, 63: 1598-1600

Zhang X L, Ha B B, Wang S J, Chen Z J, Ge J Y, Long H, He W, Da W,
Nian X M, Yi M J, Zhou X Y, Zhang P Q, Jin Y S, Bar-Yosef O, Olsen J W, Gao X. 2018. The earliest human occupation of the highaltitude Tibetan Plateau 40 thousand to 30 thousand years ago. Science, 362: 1049-1051

Zilhão J, d'Errico F. 1999. The chronology and taphonomy of the earliest Aurignacian and its implications for the understanding of Neanderthal extinction. J World Prehist, 13: 1-68

(责任编委: 陈建徽) 